\title{
ESTABILIDAD DEL PH DE CUATRO GELES A BASE DE PERÓXIDO DE HIDRÓGENO EN DISTINTOS INTERVALOS DE TIEMPO
}

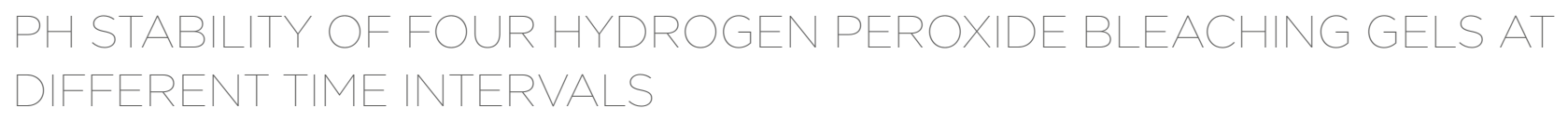

Claudia Andrea Ruiz Gamero', 2 * (i) carg1003@hotmail.com

Artículo recibido: $28 / 12 / 2020$

Arbitrado por pares

Artículo aceptado: 8/03/2021

Artículo publicado: 21/06/2021

* Autor corresponsal:

Claudia Andrea Ruiz Gamero

carg1003@hotmail.com
Citar como: Ruiz C. Estabilidad del $\mathrm{pH}$ de cuatro geles a base de peróxido de hidrógeno en distintos intervalos de tiempo. Rev Cient Odontol (Lima). 2021; 9 (2): e058.

DOI: 10.21142/2523-2754-0902-2021-058

\section{RESUMEN}

Objetivo: Comparar el $\mathrm{pH}$ de cuatro marcas de geles aclaradores a base de peróxido de hidrógeno de altas concentraciones (30\%-35\%) Whiteness HP Maxx (HPM), Lase Peroxide (LP), Whiteness HP Automixx (HPA)y Dash (DA), a través del tiempo de aplicación clínica (inicio, 15', 30’ y 45’) Materiales y métodos: El estudio fue experimental in vitro. Se evaluaron 40 muestras (dientes bovinos) divididos en 4 grupos, uno para cada marca comercial de gel. Se preparó cada gel de acuerdo con las instrucciones del fabricante y se colocó una cantidad necesaria en la superficie vestibular; posteriormente, se registró el $\mathrm{pH}$ del gel con un pHmetro digital al inicio, 15, 30 y 45 minutos. Los datos se analizaron con las pruebas Anova, Friedman y Wilcoxon. Resultados: Hubo una tendencia a la disminución del pH desde el tiempo inicial de aplicación hasta el tiempo final, con excepción del grupo de la marca DA, el cual mostró que los valores del pH fueron aumentando a través del tiempo. En la marca HPM, existe una diferencia significativa entre el tiempo inicial de aplicación del gel y el resto de tiempos. En la marca LP, a partir de los 15’ de aplicación, sí hay diferencia significativa entre los tiempos. En cuanto a la marca HPA, existieron diferencias significativas entre el tiempo inicial de aplicación y los demás tiempos. Finalmente, con la marca DA se encontró únicamente una diferencia significativa entre el tiempo inicial de aplicación y el tiempo final. Conclusiones: $\mathrm{El} \mathrm{pH}$ de los geles aclaradores disminuyó a través del tiempo de aplicación clínica en todas las marcas estudiadas, con excepción de la marca Dash 30\%, la cual mostró un aumento.

Palabras clave: aclaramiento dental, $\mathrm{pH}$, peróxido de hidrógeno

\begin{abstract}
Objective: The aim of this in vitro study was to evaluate the $\mathrm{pH}$ of four bleaching agents based on high concentration hydrogen peroxide (30-35\%) Whiteness HP Maxx (HPM), Lase Peroxide (LP), Whiteness HP Automixx (HPA) and Dash (DA) in different clinical periods (baseline, 15', 30' and 45'). Materials and methods: 40 specimens (bovine teeth) were divided into 4 groups; one group for each bleaching agent. Each bleaching agent was prepared according to the manufacturer's instructions and was applied on the vestibular surface. The $\mathrm{pH}$ of the bleaching agent was measured with a digital $\mathrm{pH}$ meter at baseline, 15, 30 and 45 minutes. ANOVA, Friedman and Wilcoxon tests were applied. Results: The $\mathrm{pH}$ values showed a trend to decreasing from the initial time of application to the final time, except for the DA group, which showed increasing $\mathrm{pH}$ values over time. The HPM group showed significant differences between baseline and the remaining periods. The LP group LP showed significant difference between $15^{\prime}$ and the other periods. The HPA group showed significant differences between baseline and the remaining periods. Finally, the DA group, showed a significant difference between baseline and 45'. Conclusions: The $\mathrm{pH}$ values of 3 of the bleaching agents decreased over time, with the exception of Dash which increased in the different time periods.
\end{abstract}

Keywords: bleaching agent, $\mathrm{pH}$, hydrogen peroxide

\footnotetext{
1 Facultad de Estomatología, Universidad Inca Garcilaso de la Vega. Lima, Perú.

2 Especialidad de Odontología Estética y Restauradora, Universidad Científica del Sur. Lima, Perú.
} 


\section{INTRODUCCIÓN}

El aclaramiento dental es un método efectivo y conservador que ha sido usado desde hace muchos años. Tiene como objetivo aclarar los dientes que presentan alteraciones de color $\left({ }^{1,2}\right)$. El color dentario depende de la translucidez del esmalte y la dentina, así como de la reflexión de los colores en el diente $\left({ }^{3}\right)$. Se forma por compuestos orgánicos que poseen cadenas de enlaces simples y dobles, que forman un cromósfero (4, $\left.{ }^{5}\right)$. El aclaramiento actúa rompiendo estos enlaces para modificar el color.

De acuerdo con la percepción individual de cada paciente, estos colores pueden no ser estéticos, por lo que buscarán mejorarlos. A través del tiempo, desde la primera vez que empezaron a desarrollarse los diferentes métodos de aclaramiento dental, se investigaron y probaron distintos materiales y técnicas con el fin de protocolizar dicho tratamiento de una manera efectiva, segura y predecible ${ }^{(6)}$.

Antes de realizar un aclaramiento dental, se debe evaluar la condición del paciente para determinar la técnica y el material a emplear. Se debe observar lo siguiente: presencia de caries dental (complejidad y ubicación); presencia de dientes oscurecidos; fisuras o cracs dentarios; descalcificaciones localizadas o manchas blancas; áreas translúcidas en bordes incisales que con el aclaramiento podrían intensificarse y crear un aspecto grisáceo, lo que afectaría el resultado final; restauraciones anteriores (se debe determinar si necesitarán retratamiento una vez finalizado el aclaramiento); simetría gingival (para optimizar el resultado final) y, por último, presencia de abrasiones, atriciones y recesiones, ya que éstas no cambiarán de color.

En cuanto al mecanismo de acción del aclaramiento, existe una teoría cromósfera $\left({ }^{1}\right)$ que se basa en la interacción del peróxido de hidrógeno con cromósferos orgánicos de la estructura dentaria. Estos cromósferos tienen áreas ricas en electrones y sus cadenas, al reaccionar, se convierten en estructuras más simples y alteran sus propiedades ópticas, lo cual disminuye la intensidad de la pigmentación $(3,5,7)$.
En este proceso se pueden distinguir 3 fases: (1) el comportamiento del agente aclarador aplicado desde la superficie externa en el esmalte; (2) la interacción de las moléculas de pigmentos con el peróxido de hidrógeno tras su penetración a la estructura dental; y (3) los cambios micromorfológicos inducidos por el peróxido en la estructura dentaria, que finalmente producen cambios ópticos. Estas tres fases dan como resultado el cambio final del color del diente después del aclaramiento.

Los efectos de los agentes aclaradores en el esmalte han sido ampliamente estudiados. Entre ellos se encuentran los cambios de la morfología, el aumento de la porosidad superficial, la exposición de prismas, la reducción del contenido orgánico, los cambios en la proporción calcio/ fosfato y la disminución de la microdureza. Todos dependerán del tiempo de permanencia del gel en el sustrato, la concentración del peróxido y el $\mathrm{pH}$ durante su uso $(5,8,11)$.

$\mathrm{E} 1 \mathrm{pH}$ de los geles aclaradores de alta concentración de $\mathrm{H}_{2} \mathrm{O}_{2}$ oscila entre 3 y $7\left({ }^{14}\right)$, lo cual indica que hay productos que tienen un $\mathrm{pH}$ crítico para el esmalte (menor a 5,5 ). $\mathrm{El} \mathrm{pH}$ de un producto aclarador tiene una relación directa con la rugosidad del esmalte una vez realizado el aclaramiento, y tiende a disminuir conforme más tiempo pase en contacto con el sustrato dentario ${ }^{8-9}$, 13-15).

Actualmente existen muchos productos de aclaramiento dental a base de peróxido de hidrógeno, que se diferencian entre sí por su concentración (la cual puede estar entre el $10 \%$ y el $40 \%$ ) y su $\mathrm{pH}$. Asimismo, algunos geles contienen otros componentes, como gluconato de calcio, fosfatos cálcicos y agentes desensibilizantes. Estos sistemas también varían en su modo de aplicación: en algunos se debe cambiar el producto cada 15 minutos $\mathrm{y}$ otros pueden permanecer por 40-50 minutos sin cambiarse.

Es importante que los productos aclaradores tengan un $\mathrm{pH}$ relativamente alcalino para minimizar los riesgos potenciales. La mayoría presenta $\mathrm{pH}$ ácido debido a que el $\mathrm{H}_{2} \mathrm{O}_{2}$ se conserva mejor en este medio, por lo que al fabricar los geles se les adiciona un ácido débil para evitar su descomposición. 
Diversos estudios reportan que el gel aclarador a base de peróxido de hidrógeno que tenga un $\mathrm{pH}$ básico será más efectivo, ya que la disociación constante del $\mathrm{H}_{2} \mathrm{O}_{2}$ será mayor que en una solución ácida $\left.{ }^{1}\right)$. También han observado que la eficacia del $\mathrm{H}_{2} \mathrm{O}_{2}$ es directamente proporcional al incremento del $\mathrm{pH}$ del gel aclarador; aunque clínicamente no es significativo en cuanto a color, sí lo es en cuanto a sensibilidad. Adicionalmente, también es importante mencionar que los geles aclaradores alcalinos usualmente muestran un $\mathrm{pH}$ más estable a través del tiempo que los ácidos $\left({ }^{1}\right)$.

El criterio del odontólogo para elegir un gel aclarador y su concentración se basa en los resultados que busca sin alterar o afectar el tejido dentario, y el tiempo que pretende invertir. Resulta de vital importancia para el odontólogo conocer a fondo las propiedades de las distintas opciones que existen actualmente, como las diferentes marcas de geles aclaradores a base peróxido de hidrógeno. Se deben conocer dichas propiedades para saber cómo se van a comportar a través del tiempo de aplicación clínica; de esta manera, se podrá establecer un protocolo de uso. Es importante establecer uno que vaya más allá de las instrucciones del fabricante (aunque sin dejar de respetarlas) y que esté de acuerdo con el tipo de peróxido y su concentración y variación de $\mathrm{pH}$. De esta manera se podrán optimizar las propiedades de cada peróxido y lograr el éxito en el tratamiento aclarador.

El objetivo de esta investigación fue comparar el $\mathrm{pH}$ de 4 marcas de geles aclaradores a base de peróxido de hidrógeno de alta concentración (30\%-35\%): Whiteness HP Maxx (HPM), Lase Peroxide (LP), Whiteness HP Automixx (HPA)y Dash (DA), a través del tiempo de aplicación clínica (inicio, 15', 30’ y 45').

\section{MATERIALES Y MÉTODOS}

El estudio fue de tipo experimental in vitro. La muestra estuvo conformada por 48 dientes bovinos y 4 geles aclaradores a base de peróxido de hidrógeno, que cumplieron con los criterios de inclusión de la investigación: dientes bovinos sanos y geles aclaradores a base de peróxido de hidrógeno con una concentración igual o mayor al 30\%.
La investigadora principal se capacitó en la correcta manipulación de los geles aclaradores a base de peróxido de hidrógeno de altas concentraciones con el Dr. Gustavo Watanabe. Además, la investigadora fue capacitada por personal de la empresa Bionet S. A. en la manipulación adecuada del pHmetro utilizado (Pasco Extech).

Fueron utilizados 4 geles aclaradores a base de peróxido de hidrógeno con una concentración del $30 \%$ a más: Whiteness HP Maxx 35\% de FGM lote 080916, con fecha de vencimiento setiembre de 2018; Lase Peroxide Sensy 35\% de DMC Lote 21754, con fecha de vencimiento setiembre de 2018; Whiteness HP Automixx 35\% de FGM Lote 271018, con fecha de vencimiento octubre de 2018, y Dash 30\% de Philips Lote DSE1001, con fecha de vencimiento diciembre de 2017.

Los 48 dientes bovinos fueron distribuidos aleatoriamente en 4 grupos, cada uno correspondiente a una marca de gel aclarador.

Para la medición del pH se utilizó un pHmetro digital marca Pasco Extech, el cual registra los valores de $\mathrm{pH}$ por segundo de contacto del electrodo del aparato con el gel de cada muestra. El pHmetro fue calibrado con soluciones básicas antes de cada experimento. Por otro lado, el electrodo fue lavado con agua destilada y secado con papel tisú entre muestra y muestra, con el fin de prevenir cualquier contaminación entre prueba y prueba. Una vez colocado el gel aclarador en cada muestra por grupo, se procedió a contactar cada una con el electrodo del pHmetro durante 5 segundos, con el fin de que este registre los valores y así poder trasladarlos a la ficha de recolección de datos en los 4 intervalos de tiempo ( 0 , 15 ', 30' y 45').

El análisis estadístico de los datos se realizó utilizando el software IBM SPSS versión 23.0. Se emplearon las pruebas no paramétricas de Friedman y Wilcoxon. Solo el grupo 4 (Dash) tuvo distribución normal, por lo que se utilizó la prueba de Anova.

Se estableció que el valor "P", para determinar la diferencia estadísticamente significativa, sería menor a 0,05 . 


\section{RESULTADOS}

En el análisis descriptivo de la variable $\mathrm{pH}$, se observó que este fue descendiendo a través de los cuatro intervalos de tiempo de aplicación clínica en 3 marcas de los geles aclaradores: Whiteness HP 35\% (tabla 1), Lase Peroxide Sensy 35\% (tabla 2) y Whiteness HP Automixx 35\% (tabla 3); sin embargo, el $\mathrm{pH}$ del gel clareador Dash (tabla 4) mostró un incremento a través del tiempo de aplicación clínica.

En la tabla 5 se comparan los diferentes pares formados entre los tiempos de aplicación clínica del gel aclarador de peróxido de hidrógeno marca Whiteness HP Maxx al 35\%. En ella se observa que existe una diferencia significativa entre T0 con T15 ( $\mathrm{P}=0,024)$, con T30 $(\mathrm{P}=0,012)$ y con $\mathrm{T} 45(\mathrm{P}=0,012)$; sin embargo, vemos que no existe esta diferencia entre T15 y T30 $(\mathrm{P}=0,660)$. Es decir, el $\mathrm{pH}$ desciende significativamente desde el tiempo inicial hasta el final (45 min), pero entre los $15 \mathrm{y}$ 30 minutos este descenso no es significativo.

En la tabla 6 se comparan de igual manera los diferentes pares formados ente los tiempos de aplicación clínica del gel aclarador marca Lase Peroxide Sensy al 35\%.

Tabla 1. Evaluación descriptiva del pH del gel aclarador Whiteness HP Automixx 35\%, Lase Peroxide 35\%, Whiteness HP Automixx 35\% y Dash 30\% al momento inicial (O') de aplicación clínica

\begin{tabular}{lcccccc}
\multicolumn{1}{c}{ GEL / TIEMPO } & \multicolumn{7}{c}{$\mathbf{0}^{\prime}$} \\
& N & Media & DE & $\boldsymbol{S}^{2}$ & Mín & Máx \\
\hline Whiteness HP Maxx 35\% & 12 & 5,99 & 0,133 & 0,018 & 5,84 & 6,27 \\
\hline Lase Peroxide 35\% & 12 & 6,34 & 0,400 & 0,160 & 5,24 & 6,73 \\
\hline Whiteness HP Automixx 35\% & 12 & 6,65 & 0,493 & 0,243 & 5,96 & 7,68 \\
\hline Dash 30\% & 12 & 5,71 & 0,404 & 0,163 & 5,20 & 6,55 \\
\hline
\end{tabular}

Tabla 2. Evaluación descriptiva del pH del gel aclarador Whiteness HP Automixx 35\%, Lase Peroxide 35\%, Whiteness HP Automixx 35\% y Dash 30\% a los 15' de aplicación clínica

\begin{tabular}{lcccccc}
\multicolumn{1}{c}{ GEL / TIEMPO } & \multicolumn{7}{c}{ I5' $^{\prime}$} & N & Media & DE & $\boldsymbol{S}^{2}$ & Mín & Máx \\
\hline Whiteness HP Maxx 35\% & 12 & 5,66 & 0,153 & 0,024 & 5,50 & 6,1 \\
\hline Lase Peroxide 35\% & 12 & 6,00 & 0,137 & 0,019 & 5,79 & 6,35 \\
Whiteness HP Automixx 35\% & 12 & 6,00 & 0,327 & 0,108 & 5,79 & 6,97 \\
\hline Dash 30\% & 12 & 6,00 & 0,532 & 0,284 & 5,19 & 6,91 \\
\hline
\end{tabular}

Se observa que no hay diferencia estadísticamente significativa entre T0 y T15 ( $\mathrm{P}=0,246)$; no obstante, a partir de los 15 minutos de aplicación, sí hay diferencia significativa entre los tiempos; $\mathrm{T} 15$ con T30 $(\mathrm{P}=0,018)$, $\mathrm{T} 15$ con T45 $(\mathrm{P}=0,012)$ y T30 con T45 $(\mathrm{P}=0,012)$. Esto quiere decir que el $\mathrm{pH}$ no desciende significativamente hasta los 15 minutos, pero que a partir de ese tiempo su descenso comienza a ser significativo entre tiempo y tiempo de aplicación.

En la tabla 7 se comparan los pares formados entre los tiempos de aplicación clínica del gel aclarador marca Whiteness HP Automixx al 35\%. Se observa que sí existen diferencias estadísticamente significativas entre el tiempo inicial de aplicación y el resto de los tiempos: T0 con T15 ( $\mathrm{P}=0,012), \mathrm{T} 0$ con T30 ( $\mathrm{P}=0,012)$, T0 con T45 ( $\mathrm{P}=0,012), \mathrm{T} 15$ con T30 $(\mathrm{P}=0,012), \mathrm{T} 15$ con T45 $(\mathrm{P}=0,012)$; sin embargo, no sucede lo mismo entre los 30 y 45 minutos de aplicación T30 con T45 (P = 0,138). Esto significa que no hay un descenso significativo del $\mathrm{pH}$ entre los 30 y 45 minutos.

En la tabla 8 se comparan los pares formados entre los tiempos de aplicación clínica del gel aclarador marca Dash al 30\%. Observamos que existe únicamente una

Tabla 3. Evaluación descriptiva del pH del gel aclarador Whiteness HP Automixx 35\%, Lase Peroxide 35\%, Whiteness HP Automixx 35\% y Dash 30\% a los 30' de aplicación clínica

\begin{tabular}{lcccccc}
\multicolumn{1}{c}{ GEL / TIEMPO } & \multicolumn{6}{c}{$\mathbf{3 0}^{\prime}$} \\
& N & Media & DE & $\mathbf{S}^{2}$ & Mín & Máx \\
\hline Whiteness HP Maxx 35\% & 12 & 5,55 & 0,074 & 0,005 & 5,47 & 5,67 \\
\hline Lase Peroxide 35\% & 12 & 5,74 & 0,081 & 0,007 & 5,62 & 5,93 \\
Whiteness HP Automixx 35\% & 12 & 5,67 & 0,198 & 0,039 & 5,34 & 6,18 \\
\hline Dash 30\% & 12 & 6,07 & 0,366 & 0,134 & 5,64 & 6,79 \\
\hline
\end{tabular}

Tabla 4. Evaluación descriptiva del pH del gel aclarador Whiteness HP Automixx 35\%, Lase Peroxide 35\%, Whiteness HP Automixx 35\% y Dash 30\% a los 45' de aplicación clínica

\begin{tabular}{lcccccc}
\multicolumn{1}{c}{ GEL / TIEMPO } & \multicolumn{6}{c}{$\mathbf{4 5}^{\prime}$} \\
& N & Media & DE & $\mathbf{S}^{\mathbf{2}}$ & Mín & Máx \\
\hline Whiteness HP Maxx 35\% & 12 & 5,46 & 0,089 & 0,008 & 5,30 & 5,61 \\
\hline Lase Peroxide 35\% & 12 & 5,62 & 0,078 & 0,006 & 5,54 & 5,79 \\
\hline Whiteness HP Automixx 35\% & 12 & 5,60 & 0,149 & 0,022 & 5,47 & 5,96 \\
\hline Dash 30\% & 12 & 6,26 & 0,300 & 0,090 & 5,82 & 6,70 \\
\hline
\end{tabular}


Tabla 5. Comparación del pH en los diferentes tiempos de aplicación clínica del gel aclarador FGM Whiteness HP $35 \%$

\begin{tabular}{|c|c|c|c|c|}
\hline \multicolumn{5}{|c|}{ SIGNIFICANCIA ESTADÍSTICA WHITENESS HP MAXX 35\% } \\
\hline \multirow[t]{2}{*}{ Tiempos } & \multirow[b]{2}{*}{ Media \pm (DE) } & \multicolumn{3}{|c|}{ Pares } \\
\hline & & PI & & $\mathbf{P 2}$ \\
\hline & & & TO VS TI5 & $0,024 * *$ \\
\hline \multirow[t]{2}{*}{ TO } & $5,99 \pm 0,133$ & & & \\
\hline & & & TO VS T30 & $0,012 * *$ \\
\hline \multirow[t]{2}{*}{ TI5 } & $5,66 \pm 0,153$ & & & \\
\hline & & $p<0,00$ I & TO VS T45 & $0,012 * *$ \\
\hline \multirow[t]{2}{*}{ T30 } & $5,55 \pm 0,074$ & & & \\
\hline & & & T30 VS TI5 & $0,660 * *$ \\
\hline \multirow[t]{3}{*}{ T45 } & $5,46 \pm 0,089$ & & & \\
\hline & & & T45 VS TI5 & $0,030 * *$ \\
\hline & & & T45 VS T30 & $0,018^{* *}$ \\
\hline
\end{tabular}

*Prueba de Friedman

**Prueba de Wilcoxon con corrección de Bonferroni

Tabla 6. Comparación del pH en los diferentes tiempos de aplicación clínica del gel aclarador DMC Lase Peroxide $35 \%$

\begin{tabular}{|c|c|c|c|c|}
\hline & SIGNIFICANCIA & TADÍSTICA & PEROXIDE 35 & \\
\hline Tiempos & & & Pares & \\
\hline & Media $\pm(D E)$ & PI & & P2 \\
\hline & & & TO VS TI5 & $0,246 * *$ \\
\hline TO & $6,34 \pm 0,400$ & & & \\
\hline & & & T0 VS T30 & $0,048 * *$ \\
\hline TI5 & $6,00 \pm 0,137$ & & & \\
\hline & & $p<0,00$ ।* & T0 VS T45 & $0,024 * *$ \\
\hline T30 & $5,74 \pm 0,081$ & & & \\
\hline & & & T30 VS TI5 & $0,0 \mid 8 * *$ \\
\hline T45 & $5,62 \pm 0,078$ & & & \\
\hline & & & T45 VS TI5 & $0,012 * *$ \\
\hline & & & T45 VS T30 & $0,0 \mid 2 * *$ \\
\hline
\end{tabular}

*Prueba de Friedman

**Prueba de Wilcoxon con corrección de Bonferroni

diferencia significativa estadísticamente entre el tiempo inicial de aplicación y el tiempo final T0 con T45 $(\mathrm{P}=0,006)$, los demás tiempos no muestran mayores diferencias entre sí, T0 con T15 $(\mathrm{P}=0,519), \mathrm{T} 0$ con T30 $(\mathrm{P}=0,212), \mathrm{T} 15$ con $\mathrm{T} 30(\mathrm{P}=1,000), \mathrm{T} 15$ con $\mathrm{T} 45$ $(\mathrm{P}=0,750)$ y $\mathrm{T} 30$ con $\mathrm{T} 45(\mathrm{P}=1,000)$. Es decir, en este caso, el $\mathrm{pH}$ ha aumentado significativamente desde el tiempo inicial comparándolo con el tiempo final de aplicación.
Tabla 7. Comparación de significancia estadística de la variación del pH en los diferentes tiempos de aplicación clínica del gel aclarador FGM Whiteness HP Automixx 35\%

\begin{tabular}{|c|c|c|c|c|}
\hline \multicolumn{5}{|c|}{ SIGNIFICANCIA ESTADÍSTICA WHITENESS HP AUTOMIXX 35\% } \\
\hline \multirow[t]{2}{*}{ Tiempos } & \multirow[b]{2}{*}{ Media \pm (DE) } & \multicolumn{3}{|c|}{ Pares } \\
\hline & & PI & & P2 \\
\hline & & & TO VS TI5 & $0,012 * *$ \\
\hline \multirow[t]{2}{*}{ TO } & $6,65 \pm 0,493$ & & & \\
\hline & & & TO VS T30 & $0,012 * *$ \\
\hline \multirow[t]{2}{*}{ TI5 } & $6,00 \pm 0,327$ & & & \\
\hline & & $\mathrm{p}<0,00$ I* & TO VS T45 & $0,012 *$ \\
\hline \multirow[t]{2}{*}{ T30 } & $5,67 \pm 0,198$ & & & \\
\hline & & & T30 VS TI5 & $0,012 * *$ \\
\hline \multirow[t]{3}{*}{ T45 } & $5,60 \pm 0,149$ & & & \\
\hline & & & T45 VS TI5 & $0,012 * *$ \\
\hline & & & T45 VS T30 & $0,138 * *$ \\
\hline
\end{tabular}

*Prueba de Friedman

**Prueba de Wilcoxon con corrección de Bonferroni

Tabla 8. Comparación del pH en los diferentes tiempos de aplicación clínica del gel aclarador Philips Dash 30\%

\begin{tabular}{|c|c|c|c|c|}
\hline \multicolumn{5}{|c|}{ SIGNIFICANCIA ESTADÍSTICA DASH 30\% } \\
\hline \multirow[t]{2}{*}{ Tiempos } & & & Pares & \multirow[b]{2}{*}{$\mathbf{P 2}$} \\
\hline & Media $\pm(D E)$ & PI & & \\
\hline & & & TO VS TI5 & $0,519 * *$ \\
\hline \multirow[t]{2}{*}{ TO } & $5,7 \mid \pm 0,404$ & & & \\
\hline & & & T0 VS T30 & $0,212 * *$ \\
\hline \multirow[t]{2}{*}{ TI5 } & $6,00 \pm 0,532$ & & & \\
\hline & & $0,016(p>0.001)$ & T0 VS T45 & $0,006 * *$ \\
\hline \multirow[t]{2}{*}{ T30 } & $6,07 \pm 0,366$ & & & \\
\hline & & & T30 VSTI5 & $1,00 * *$ \\
\hline \multirow[t]{3}{*}{$\mathrm{T} 45$} & $6,26 \pm 0,300$ & & & \\
\hline & & & T45 VS TI 5 & $0,750 * *$ \\
\hline & & & T45 VS T30 & $1,00 * *$ \\
\hline
\end{tabular}

*Prueba de Anova

**Prueba de Bonferroni

\section{DISCUSIÓN}

El propósito de este estudio fue evaluar la estabilidad del $\mathrm{pH}$ de cuatro geles aclaradores a base de peróxido de hidrógeno (Whiteness HP Maxx 35\% FGM, Lase Peroxide Sensy 35\% DMC, Whiteness HP Automixx 35\% FGM y Dash 30\% Philips) en cuatro intervalos de tiempo: 0', 15', 30'y 45'. 

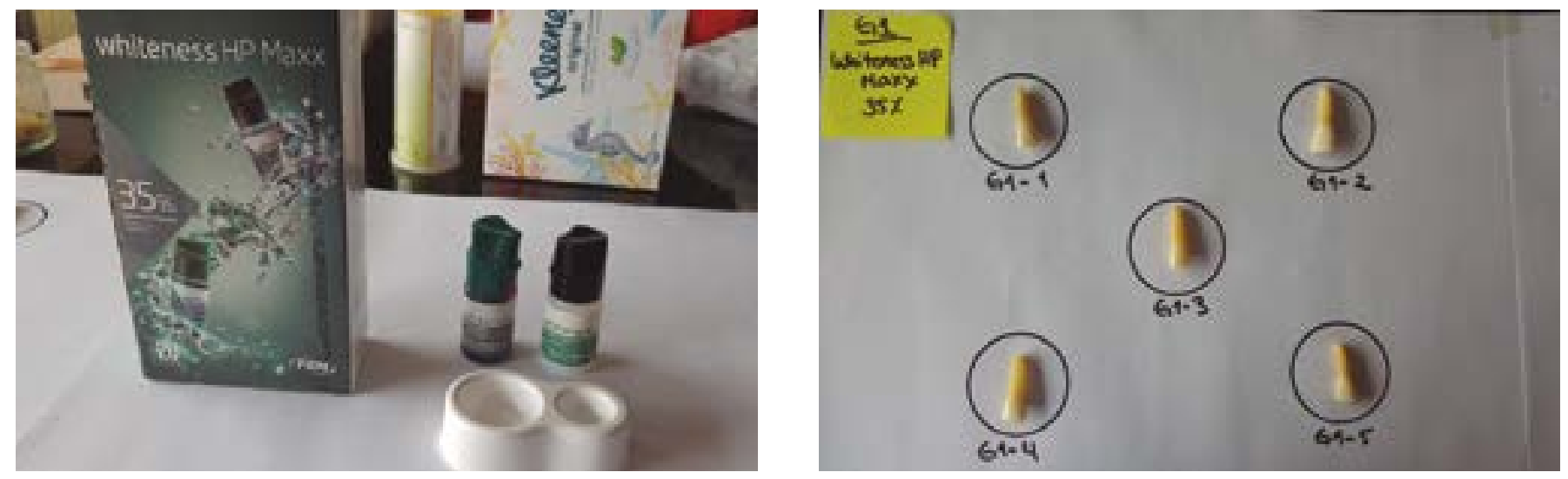

Distribución aleatoria de las muestras en el Grupo G1; Whiteness HP Maxx 35\%.
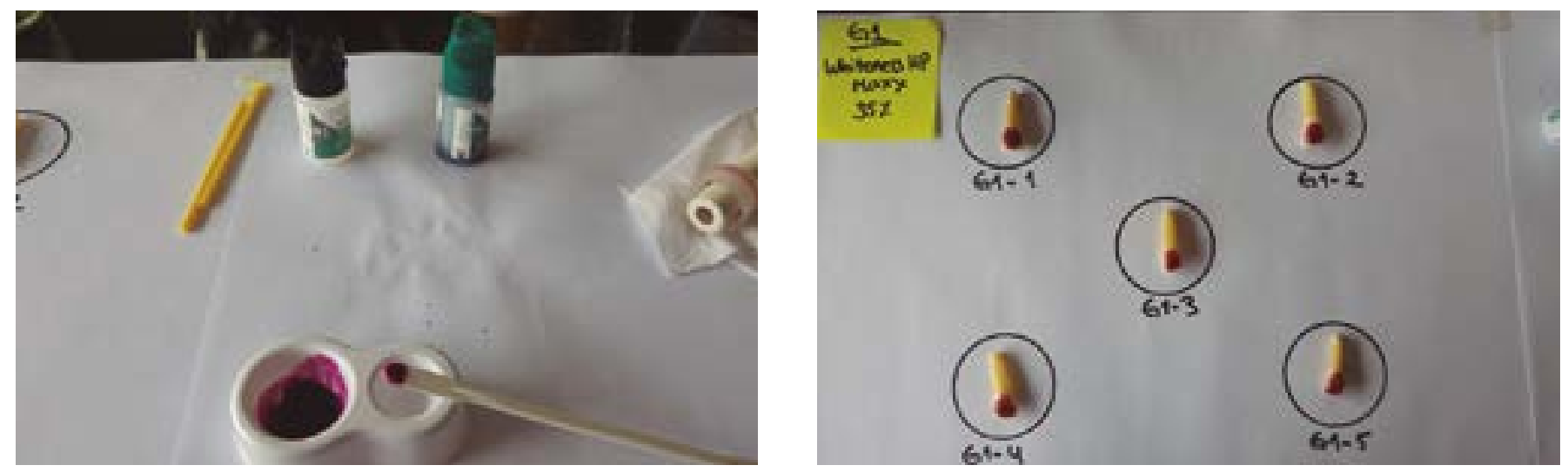

Mezcla, activación y aplicación del gel según indicaciones del fabricante.

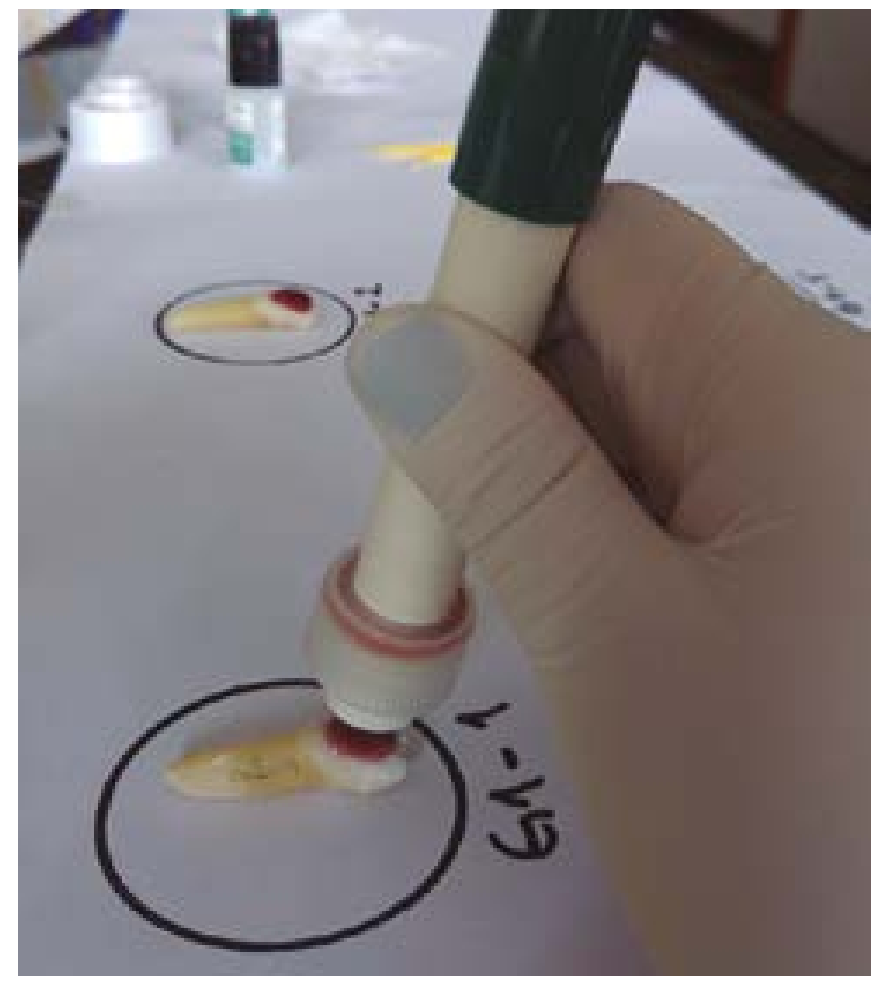

Una vez colocado el gel en cada muestra se procedió a contactar cada muestra del G1 con el pHmetro durante 05 segundos con el fin de que éste registre los valores y poder trasladarlos a la ficha de recolección de datos a los $0^{\prime}, 15^{\prime} 30^{\prime}$ y $45^{\prime}$.

Figura I. Secuencia de pasos por grupo de trabajo 
Se evidenció una disminución del $\mathrm{pH}$, con diferencia significativa entre algunos intervalos de tiempo de los geles Whiteness HP Maxx 35\%, Lase Peroxide Sensy 35\% y Whiteness HP Automixx 35\%, por lo tanto, la hipótesis nula fue rechazada. En el caso del gel aclarador Dash 30\% se evidenció, por el contrario, un aumento del $\mathrm{pH}$ que fue significativo solo entre el tiempo inicial (T0) y el final (T45).

Muchos de los productos aclaradores presentan valores bajos de $\mathrm{pH}$ para asegurar la estabilidad del peróxido de hidrógeno (1). Como ya se dijo, se observó que hubo una tendencia a la disminución del $\mathrm{pH}$. Estos resultados son similares a los del estudio de Price et al. (2000), que demostraron que los geles aclaradores a base de peróxido de hidrógeno pueden mostrar valores bajos de $\mathrm{pH}$ e ir disminuyendo progresivamente a través del tiempo.

Diversos estudios han evaluado el $\mathrm{pH}$ de distintos geles aclaradores $(10,12,14,15)$ ya que este es considerado un factor muy importante por ser el que determina el rango de acción del proceso aclarador; mientras mayor sea el $\mathrm{pH}$, se producirán más radicales libres $\left({ }^{10}\right)$. Por otro lado, el nivel de $\mathrm{pH}$ de un agente aclarador se relaciona directamente con las probables alteraciones micromorfológicas que sufriría el esmalte durante un aclaramiento dental (8). Se sabe que el $\mathrm{pH}$ crítico para el esmalte es de 5,5 $\left({ }^{13}\right)$, y muchos de los geles aclaradores que existen muestran que están por debajo de ese rango $\left({ }^{14}\right)$. La presente investigación evidenció que los cuatro agentes evaluados no tenían un valor menor a 5,5 durante los cuatro intervalos de tiempo considerados, pero sí mostraron valores muy cercanos, por lo que es válido mencionar que la preservación del producto es igual de importante que la preservación de la estructura dentaria para los fabricantes.

Sun et al. (2011) mostraron que tanto los productos con $\mathrm{pH}$ ácido como con $\mathrm{pH}$ neutro, tuvieron la misma eficacia en el aclaramiento; sin embargo, los geles con pH ácido causaron un 30\% más de efectos adversos en la superficie del esmalte dental. Ese hallazgo indica que la desmineralización de la superficie del esmalte parece estar causada principalmente por el $\mathrm{pH}$ bajo en lugar de que sea por el peróxido de hidrógeno en sí $\left({ }^{39}\right)$.
Los promedios del $\mathrm{pH}$ por intervalos de tiempo y por marcas encontrados en este estudio, si bien no son críticos para el esmalte y además fueron tomados de dientes bovinos como muestra, al estar cerca de ese nivel crítico invita a tomar en cuenta que en la parte clínica ese aspecto podría relacionarse con la sensibilidad que existe durante un aclaramiento. Arana (2013) menciona que la desmineralización de la superficie del esmalte causada por el $\mathrm{pH}$ bajo de los agentes aclaradores podría tener un efecto sobre la actividad biodinámica de los mismos, lo que aumenta los procesos erosivos; clínicamente, el aumento de la porosidad permite que el agente aclarador penetre más fácilmente a través del esmalte y la dentina hacia la pulpa produciendo una injuria a nivel pulpar que se traduciría en la sensibilidad por aclaramiento dental $\left({ }^{13}\right)$.

Ha sido reportado por Price et al. (2000) que mientras más elevada sea la concentración del peróxido de hidrógeno de un gel, más ácido será el $\mathrm{pH}\left({ }^{14}\right)$. Como se recuerda, la concentración del gel aclarador Dash es del 30\%, menor a las concentraciones del resto de marcas (35\%), y fue el único grupo que tuvo un incremento de los valores del $\mathrm{pH}$ a través del tiempo de aplicación clínica; esto puede deberse a que, dentro de su composición, además de una menor concentración de peróxido de hidrógeno, presenta hidróxido de amonio $\left(\mathrm{NH}_{4} \mathrm{OH}\right)$, el cual es una solución de amoniaco en agua que tiene propiedades alcalinas y se usa como regulador del $\mathrm{pH}$. Price et al., en su estudio, también afirman que la liberación de amonio (o hidroxilo de amonio) eleva el $\mathrm{pH}$ del gel aclarador a los 15 minutos, con lo que es capaz de alcalinizarlo $\left({ }^{14}\right)$. Esto coincide con los resultados del presente estudio, que muestra que el grupo Dash $30 \%$ a los 15 minutos ya mostraba valores más elevados que al inicio de la aplicación.

Otro estudio que presentó resultados similares fue el de Trentino et al. (2015), quienes evaluaron el $\mathrm{pH}$ de 7 marcas de geles aclaradores a base de peróxido de hidrógeno con diferentes concentraciones: Whiteness HP Maxx 35\%, Lase Peroxide 35\%, Lase Peroxide II 25\%, Lase Peroxide Lite 15\%, Whitegold Office 35\%, Whiteness HP Blue Calcium 35\% y Whiteness HP Blue Calcium 20\%. Utilizaron un pHmetro digital Sentron y registraron los valores de $\mathrm{pH}$ de cada muestra 
a los 30 segundos de aplicación en la superficie del diente bovino (tiempo inicial) y 30 segundos después de que se cumpla el tiempo de aplicación del gel (tiempo final). Los resultados mostraron que los valores de $\mathrm{pH}$ de los geles aclaradores tendieron a disminuir a través del tiempo inicial al final, con la excepción de las marcas Whitegold Office 35\% y Whiteness HP Blue Calcium $35 \%$, las cuales mostraron valores más altos de $\mathrm{pH}$ en el tiempo final $\left({ }^{10}\right)$.

Actualmente, la mayoría de los agentes aclaradores a base de peróxido de hidrógeno aún presentan valores ácidos de $\mathrm{pH}$ con el fin de que dicho componente se mantenga estable y no se descomponga muy rápidamente $(2,3)$, y además que su composición también consta de ingredientes como glicerina, sales, saborizantes, entre otros, los cuales podrían alterar o manipular sus $\mathrm{pH}$ a través del tiempo desde su activación. Por tanto, es importante continuar estudiando los efectos que estos componentes pueden tener no solo en el comportamiento del peróxido de hidrógeno, sino también en el $\mathrm{pH}$ del gel aclarador, ya que finalmente estos cambios podrían afectar al tejido dentario en un determinado tiempo de aplicación clínica.

Por otro lado, es importante mencionar también que, al realizar un aclaramiento dental, la temperatura intraoral puede afectar los niveles de $\mathrm{pH}$ de agentes aclaradores durante el procedimiento clínico, por lo que es necesario realizar investigaciones in vivo sobre este tema.

Se sugiere, en próximos estudios, evaluar el $\mathrm{pH}$ de un mayor número de geles aclaradores y, en lo posible, realizarlo en pacientes, según las instrucciones del fabricante en cuanto a manipulación y tiempos de exposición con el fin de determinar el comportamiento del $\mathrm{pH}$ en el medio oral y las variaciones que puede tener.

\section{CONCLUSIONES}

- Existió una tendencia a la disminución del pH de los geles aclaradores a través del tiempo de aplicación clínica en todas las marcas estudiadas, con excepción de la marca Dash 30\%, la cual mostró un aumento.
- El pH de la marca Whiteness HP Maxx 35\% descendió significativamente desde el tiempo inicial hasta el final (45 min), pero entre los 15 y 30 minutos este descenso no es significativo.

- En el gel aclarador Lase Peroxide 35\%, el pH no desciende significativamente hasta los 15 minutos, pero a partir de ese tiempo su descenso comienza a ser significativo entre tiempo y tiempo de aplicación.

- En la marca Whiteness HP Automixx 35\% existen diferencias estadísticamente significativas entre el $\mathrm{pH}$ del tiempo inicial de aplicación y el resto de los tiempos; sin embargo, no sucede lo mismo entre los 30 y 45 minutos de aplicación. Esto significa que no hay un descenso significativo del $\mathrm{pH}$ en el lapso mencionado.

- La marca Dash 30\% muestra una diferencia significativa entre el tiempo inicial de aplicación y el tiempo final (45 min), el pH aumentó desde el tiempo inicial al compararlo con el tiempo final de aplicación.

Agradecimientos: Al biólogo Jorge Muñoz, por el apoyo brindado al proporcionar los contactos necesarios para la adquisición del equipo necesario para este estudio.

Contribución autoría: Claudia Andrea Ruiz Gamero ha participado en la concepción, la recopilación de información, el análisis y la interpretación de los datos, así como la redacción y aprobación de la versión final del artículo.

Fuente de financiamiento: Autofinanciado.

Conflicto de intereses: La autora declara no tener ningún conflicto de intereses. 


\section{REFERENCIAS BIBLIOGRÁFICAS}

1. Perdigao J, editor. Tooth Whitening: An evidencebased perspective. Suiza: Springer; 2016. doi: https://doi. org/10.1007/978-3-319-38849-6

2. Berga A, Forner L, Amengual J. Blanqueamiento vital domiciliario: comparación de tratamientos con peróxido de hidrógeno y peróxido de carbamida. Med Oral Patol Oral Cir Bucal. 2006; 11: E94-9.

3. Munther A. An overview of tooth-bleaching techniques: chemistry, safety and efficacy. Periodontology 2000. 2008; 48:48169. doi: https://doi.org/10.1111/j.1600-0757.2008.00258.x

4. Wille T, Combe E, Pesun I, Giles D. Rheological characteristics of tooth bleaching materials. Journal of Oral Rehabilitation. 2000; 27: 1060-3. doi: https://doi.org/10.1111/j.13652842.2000.00647.x

5. Abe A, Youssed M, Turbino M. Effect of bleaching agents on the nanohardness of tooth enamel, composite resin, and the tooth-restoration interface. Operative Dentistry. 2015; 40-6. doi: https://doi.org/10.2341/14-153-1

6. Abouassi T, Wolkewitz M, Hahn P. Effect of carbamide peroxide and hydrogen peroxide on enamel surface: an in vitro study. Suiza: Springer; 2010. doi: https://doi.org/10.1007/s00784-0100439-1

7. Ran S, Wertz P. Review of the mechanism of tooth whitening. J Esthet Restor Dent. 2015; 27 (5): 240-57. doi: https://doi. org/10.1111/jerd.12152

8. Barbosa A. Effect of $\mathrm{pH}$ on whitening efficacy of $35 \%$ hydrogen peroxide and enamel microhardness. J Esthet Restor Dent. 2018; 1-6. doi: https://doi.org/10.1111/jerd.12367

9. Torres C. Influence of concentration and activation on hydrogen peroxide diffusion through dental tissues in vitro. The Scientific World Journal. 2013; 1-5. doi: https://doi. org/10.1155/2013/193241

10. Trentino A, Soares A, Hungaro M, Kiyoshi S, Mondelli R. Evaluation of $\mathrm{pH}$ levels and surface roughness after bleaching and abrasion tests of eight commercial products. Photomedicine and laser surgery. 2015; 33(7): 372-7. doi: https://doi.org/10.1089/ pho.2014.3869

11. Bistey T, Nagy I, Simó A, Hegedus C. In vitro FT-IR study of the effects of hydrogen peroxide on superficial tooth enamel. J Dent. 2007; 35: 325-30. doi: https://doi.org/10.1016/j. jdent.2006.10.004

12. Soares A, Bombonatti J, Alencar M, Consolmagno E, Honorio $\mathrm{H}$, Mondelli R. Influence of $\mathrm{pH}$, bleaching agents, and acid etching on surface wear of bovine enamel. J Appl Oral Sci. 2016; 24 (1): 24-30. doi: https://doi.org/10.1590/1678-775720150281

13. Arana L. La importancia del pH en los agentes aclaradores. Dental Tribune Latinoamérica. 2013; 15-8.

14. Price R, Sedarous M, Hiltz G. The $\mathrm{pH}$ of tooth-whitening products. J Can Dent Assoc. 2000; 66: 421-6.

15. Marson F, Sensi L, Reis R. Novo conceito na clareacao dentaria pela tecnica no consultorio. R Dental Press Estét. 2008; 5 (3): $55-66$.
16. Al-Qunaian T, Matis B, Cochran M. In vivo kinetics of bleaching gel with three-percent hydrogen peroxide within the first hour. Operative Dentistry. 2003; 28 (3): 236-41.

17. Munther A. An overview of tooth-bleaching techniques: chemistry, safety and efficacy. Periodontology 2000. 2008; 48: 148-69. doi: https://doi.org/10.1111/j.1600-0757.2008.00258.x

18. Browning W. Power bleaching.Journal Compilation.2011; 23 (1): 61-3. doi: https://doi.org/10.1111/j.1708-8240.2010.00390.x

19. Matis B, Cochran M, Eckert G. Review of the effectiveness of various tooth whitening systems. Operative Dentistry. 2009; 34 (2): 230-5. doi: https://doi.org/10.2341/08-74

20. Cavalli V, Arrais A, Giannini M, Ambrosano G. Highconcentrated carbamide peroxide bleaching agents effects on enamel surface. J Oral Rehabil. 2004; 31 (2): 155-9. doi: https:// doi.org/10.1111/j.1365-2842.2004.01138.x

21. Dahl J. Tooth bleaching - A Critical Review of the Biological Aspects. Crit. Rev. Oral. Biol. Med. 2003; 14 (4): 292-304. doi: https://doi.org/10.1177/154411130301400406

22. Riehl H. Consideraciones clínicas sobre terapias de clareamiento dental. Scientific-A. 2007; 1 (1): 68-78.

23. Joiner $A$. The bleaching of teeth: A review of the literature. J Dent. 2006; 34: 413-9. doi: https://doi.org/10.1016/j. jdent.2006.02.002

24. Karpinia K, Magnusson I, Barker M, Gerlach R. Clinical comparison of two-self-directed bleaching systems. Journal of Prosthodont. 2003; 12: 242-8. doi: https://doi.org/10.1016/ s1059-941x(03)00102-5

25. Lee G, Lee M, Lum R, Poh R, Lim K. Extraradicular diffusion of hydrogen peroxide and $\mathrm{pH}$ changes associated with intracoronal bleaching of discoloured teeth using different bleaching agents. Int Endod J. 2004; 37: 500-6. doi: https://doi.org/10.1111/ j.1365-2591.2004.00838.x

26. Toldberg M, Grootveld, Lynch E. Undesirable and adverse effects of tooth whitening products: a review. Clin Oral Invest. 2010; 14: 1-10. doi: https://doi.org/10.1007/s00784-009-0302-4

27. Shethri S, Matis B, Cochran M,Zenokis R, Stropes M. A clinical evaluation of two in-office bleaching products. Oper Dent. 2003; 28 (5): 488-95. doi: https://doi.org/10.2341/08-64

28. Sulieman M, Addy M, MacDonald E, Rees J. The effect of hydrogen peroxide concentration on the outcome of tooth whitening: an in vitro study. J Dent. 2004; 32: 295-9. doi: https:// doi.org/10.1016/j.jdent.2004.01.003

29. Tredwin C, Nesik S. Hydrogen peroxide tooth-whitening products: Review of adverse effects and sofety issues. British Dental Journal. 2006; 200 (7). doi: https://doi.org/10.1038/ sj.bdj.4813423

30. Noblom L. Estudio de cuatro sistemas de blanqueamiento dental, en clínica y ambulatorio. 2008. Disponible en: http:// www.clinicaeco.com/blog/wpcontent/uploads/2008/05/ elblanqueamiento-ddental-estado-de-la-cuestion.pdf

31. Pelkova M. Efectos clínicos y estructurales del blanqueamiento dental. Odontol sanmarquina. 2005; 8 (2): 34-6. doi: https://doi. org/10.15381/os.v8i2.3145 
32. Kohen F, De Franceschi C, Rodríguez G. Color, estética y blanqueamiento integrados: Atlas de procedimientos y técnicas. Buenos Aires: Sacerdoti; 2007.

33. Deliperi S, Bardwell D, Papathanasiou A. Clinical evaluation of a combined in-office and take-home bleaching system. J Am Dent Assoc. 2004; 135: 628-34. doi: https://doi.org/10.14219/jada. archive.2004.0252

34. Vélez C, Delgado L. Henostroza G. Blanqueamiento de piezas vitales. Estética en odontología restauradora. Madrid: Ripano; 2004.

35. Matis B, Mousa H, Cochran M, Eckert G. Clinical evaluation of bleaching agents of different concentrations. Quintessence Int. 2000; 31: 303-10. doi: https://doi.org/10.2341/08-64
36. Christensen G. Are snow-white teeth really so desirable? JADA. 2005; 136: 933-5. doi: https://doi.org/10.14219/jada. archive.2005.0295

37. Efeoglu N, Wood D, Efeoglu C. Thirty-five percent carbamide peroxide application causes in vitro demineralization of enamel. Dental Materials. 2007; 23:900-4. doi: https://doi.org/10.1016/j. dental.2006.06.032

38. Matis B, Cochran M, Franco M, Al-Ammar G, Eckert G, Stropes M. Eight in-office tooth whitening systems evaluated in vivo: A pilot study. Oper Dent. 2007; 32 (4): 322-7. doi: https:// doi.org/10.2341/06-135

39. Sun L, Liang S, Sa Y, Wang Z, Ma X, Jiang T. Surface alteration of human tooth enamel subjected to acidic and neutral $30 \%$ hydrogen peroxide. J Dent. 2011; 39: 686-92. doi: https://doi. org/10.1016/j.jdent.2011.07.011 